\title{
A Three-Phase Matheuristic for the Time-effective Electric Vehicle Routing Problem with Partial Recharges
}

\author{
M. Bruglieri ${ }^{1}$ S. Mancini ${ }^{2}$ F. Pezzella ${ }^{3}$ O. Pisacane ${ }^{4}$ \\ S. Suraci ${ }^{3}$
}

\begin{abstract}
We propose a three-phase matheuristic, combining an exact method with a Variable Neighborhood Search local Branching (VNSB) to route a fleet of Electric Vehicles (EVs). EVs are allowed stopping at the recharging stations along their routes to (also partially) recharge their batteries. We hierarchically minimize the number of EVs used and the total time spent by the EVs, i.e., travel times, charging times and waiting times (due to the customer time windows). The first two phases are based on Mixed Integer Linear Programs to generate feasible solutions, used in a VNSB algorithm. Numerical results on benchmark instances show that the proposed approach finds good quality solutions in reasonable amount of time.
\end{abstract}

Keywords: Time Windows, Green Logistics, Recharging Decisions, Variable Neighborhood Search.

1 Dipartimento di Design, Politecnico di Milano, Italy.

Email: maurizio.bruglieri@polimi.it

2 Dipartimento di Matematica e Informatica, Universitá di Cagliari, Italy.

Email: simona.mancini@unica.it

${ }^{3}$ Dipartimento di Ingegneria dell'Informazione, Universitá Politecnica delle Marche, Italy.

Email: pezzella@dii.univpm.it, s.suraci@univpm.it

${ }^{4}$ Facoltá di Ingegneria, Universitá degli Studi e-Campus, Italy.

Email: ornella.pisacane@uniecampus.it 


\section{Introduction and literature review}

One objective of the European Union is to decrease the harmful emissions of about $95 \%$, by 2050. To this end, the Electric Vehicles (EVs) have a key role since they do not produce local greenhouse emissions. However, a poor driving range, a scarce presence of Recharging Stations (RSs) in the territory and high acquisition costs limit their diffusion. Therefore, optimizing their routes becomes crucial to efficiently manage those factors.

Our aim is to efficiently route a fleet of EVs, based at a single depot, to serve a set of customers. Each route has to start/end from/to the depot, respecting: the customer Time Windows (TW), the limited battery capacity and the load capacity. Two hierarchical objectives to minimize are considered. The first one is the number of EVs used $(\mu)$, while the second one is the Total Time (TT) spent by the EVs, i.e., travel times, charging times and waiting times (due to the customer TWs). A time optimization is significant in distribution networks operating in urban contexts and/or for in the last mile Logistics and it has been already considered in the VRPTW [8]. Therefore, in this paper, a time-effective Electric Vehicle Routing Problem with Time Windows is addressed, making possible also Partial Recharges (PRs) at the RSs: we refer to this problem as the E-VRPTW-PR.

The E-VRPTW, with only full recharges, is introduced in [9] where it is addressed by both Mixed Integer Linear Programming (MILP) and a hybrid meta-heuristic. Also in this work, two hierarchical objectives are minimized: $\mu$ and then, the total travel distance. In [1], the E-VRPTW-PR is introduced and modeled from a time effective perspective, making the battery level, reached at each RS, a decision variable. For it, a Variable Neighborhood Search Branching (VNSB) is designed. Variables and constraints of the MILP model are decreased, expressing the TT as sum of the differences between the ending and the starting time of each EV [2], cloning the depot into two sets. The advantages of the PRs and of using different recharge technologies are investigated in [4].

Our main contributions are: a Three-Phase Matheuristic (TPM) for the E-VRPTW-PR; a novel initialization procedure; a methodology competitive with the ones of the literature on small size benchmark instances and strongly outperforming them on medium size ones. 


\section{Problem Description}

The E-VRPTW-PR is modeled on a directed graph $G=(V, A)$ where $V$ contains the set of customers $N$, the set of RSs $F$ and the set of depots $D_{0}$ and $D_{0^{\prime}}$, used at the beginning and at the end of each route, respectively. The set $F$ contains the clones of the RSs for allowing passing through them more than once. The depot $(0)$ is cloned into $D_{0}$ and $D_{0^{\prime}}$ for distinguishing the starting and the ending time of each route. For simplicity, $V^{\prime}=V \backslash D_{0^{\prime}}$ and $V^{\prime \prime}=V \backslash D_{0}$ are used. The set $A$ contains the arcs between all the pairs of nodes except the ones between two depots or the same clones of a RS. For each of them, the kilometer distance $d_{i j}$ and the travel time $t_{i j}$ are known while, for each $i \in N$, the pick-up demand $q_{i} \geq 0$, the time window $\left[e_{i}, l_{i}\right]$ and the service time $s_{i} \geq 0$ are given. The interval $\left[e_{0}, l_{0}\right]$ specifies the earliest departure time from 0 and the latest arrival time to 0 such that: $\forall i \in D_{0},\left[e_{0},+\infty\right]$, while $\forall i \in D_{0^{\prime}},\left[0, l_{0}\right]$. The load capacity $C$ and the battery capacity $Q$ are known for each EV as well as the battery consumption $r$ and the recharging rate $g$. Each EV: starts from a node in $D_{0}$, ends to a node in $D_{0^{\prime}}$ and respects both the cargo and battery capacity. Intermediate stops at RSs for a (eventually partial) recharges are allowed. The recharging time $\rho$ is linearly proportional by $g$ to the recharged level (as assumed in [9]). The goal is to minimize firstly $\mu$ and then, TT, conveniently expressed as sum of the differences between the arrival time to 0 and the departure time from 0 of each route. The constraints are not exceeding the load capacity and that the routes have to take into account the remaining battery level.

\section{A Three-Phases Matheuristic}

This section describes a matheuristic consisting of three phases, sequentially applied, for solving the E-VRPTW-PR:

- Phase 1: A feasible solution $S^{1}$, consisting of a set of routes, is found by solving the MILP model of the Green Capacitated VRPTW (G-CVRPTW) that extends the one of [3], for the GVRP, including TWs and capacity constraints. TT is minimized. Unlike the E-VRPTW-PR, $\mu$ is limited to $M$ by adding a constraint as well as TT that cannot exceed a maximum route duration $\left(l_{0}\right)$. The advantage of solving a G-CVRP-TW is that, minimizing TT, we do not need to explicitly express the starting and the arrival times from/to the depot. The constraint on the route duration is satisfied by calculating only the arrival times to the nodes (excluding 0 ), without cloning the depot. The value of $\rho$ does not depend on the residual battery level and 
the G-CVRP-TW is extremely easier than our original problem. Moreover, the following property holds: choosing a proper $\rho$, each feasible solution of the G-CVRP-TW is feasible also for our problem. To properly set $\rho$, let us consider the worst case, i.e., when the battery level is zero and a full recharge is required. In such a case, $\rho=\frac{Q}{g}$.

- Phase 2: $S^{1}$ is an input of the model of the E-VRPTW-PR ([1]). To maintain the same routes of $S^{1}$, the arc variables $x_{i j}$ are fixed to the values in $S^{1}$. The resulting model optimizes the starting time at the depot (then, the arrival times at nodes) and the battery charge level reached at each RS (then, the battery level at nodes).

- Phase 3: The solution $S^{2}$ of Phase 2 is the starting point of the VNSB (VNS and local Branching) of [2]. The VNS adds linear constraints to the original problem for systematically changing the neighborhoods ([6], [7]). A local branching can be performed by adding linear constraints to MILP formulation, modeling the Hamming Distance (HD) based neighborhoods ([5]). The solutions of a neighborhood, within a radius $r h s$, are those with $H D \leq r h s$. By a Large Neighborhood Search (LNS), neighborhoods are sequentially analyzed, with $r h s \in\left[r h s_{\text {min }}^{L}, r h s_{\text {max }}^{L}\right]$ and a step of $k s t e p^{L}$. Each neighborhood is defined by adding to the model a constraint stating that the HD between a solution of the neighborhood and the current one must be lower than or equal to rhs. This model is solved by CPLEX, in a time limit $T L^{L}$ and keeping the best solution obtained. In case of no improvement, $r h s=r h s+k s t e p^{L}$; otherwise, the new solution found is kept as the best and $r h s=r h s_{\min }^{L}$. In case of no improvement and $r h s=r h s_{\text {max }}^{L}$, LNS stops. Then, a shaking phase evaluates disjointed neighborhoods, represented by a ring of $r h s_{\min }^{S}+k<r h s<r h s_{\min }^{S}+k+$ $k s t e p^{S}$, with an initial $k=0$. The resulting model is solved by CPLEX within a time limit of $T L^{S}$. The first feasible solution found is kept as the new current one and from it, the LNS restarts. In case of no feasible solution within $T L^{S}, k$ is incremented by $k s t e p^{S}$ and the procedure iterates until $r h s_{\min }^{S}+k+k s t e p^{S} \geq r h s_{\max }^{S}$. The Phase 3, and thus the whole matheuristic, ends when a given total CPU time limit is reached.

The MILP model of C-GVRP-TW (Phase 1) is formulated through binary variables $x_{i j}$ equal to 1 if arc is used, 0 otherwise, $\forall(i, j) \in A$ and continuous variables $y_{j}$ representing the battery level upon arrival at $j, \tau_{j}$ for the arrival time at $j$ and $u_{j}$ for the remaining cargo capacity at $j, \forall j \in V$. 


$$
\begin{gathered}
\min \sum_{i \in V} \sum_{j \in V} t_{i j} x_{i j} \\
\sum_{j \in V j \neq i} x_{i j}=1 \quad \forall i \in N \\
\sum_{j \in V j \neq i} x_{i j} \leq 1 \quad \forall i \in F \\
\sum_{i \in V i \neq j} x_{j i}=\sum_{i \in V i \neq j} x_{i j} \quad \forall j \in N \cup F \\
\sum_{j \in V j \neq 0} x_{0 j} \leq M \\
\sum_{j \in V j \neq 0} x_{j 0} \leq M \\
\left.y_{j} \leq y_{i}-r \cdot t_{i j}+s_{j}\right) x_{i j}-l_{0}\left(1-x_{i j}\right) \quad \forall i \in V, j \in N \cup F, i \neq j \\
\left.y_{j}=Q \quad \forall j \in F \cup x_{i j}\right) \quad \forall j \in N, i \in N \cup F, i \neq j \\
y_{i} \geq r \cdot t_{i j} x_{i 0} \quad \forall i, j \in N \cup F \\
e_{i} \leq \tau_{i} \leq l_{i} \quad \forall j \in V \backslash\{F\} \\
q_{i} x_{i j}+C\left(1-x_{i j}\right) \quad \forall j \in V, i \in V, i \neq j \\
x_{i j} \in\{0,1\} \quad \forall i \in V, \forall j \in V \\
y_{j} \geq 0 \quad \forall i \in N \cup F
\end{gathered}
$$

Function (1) is the minimization of TT. Each customer is visited exactly once (2) while a RS may be visited only once (3). Route continuity is ensured by (4) while the number of routes is limited by (5)-(6). Arrival time at a node is tracked by (7) and the battery charge level, upon arrival at a node, is ruled by (8). Sub-tours are excluded by time (7) and battery charge level (8) tracking constraints. Battery charge is $Q$ at the departure from the depot and reset up to $Q$ upon each visit to a RS (9). After visiting the last node in a route, remaining battery charge level allows returning to 0 (10). Each time windows is respected and an EV returns before the depot closure (11). Capacity and variable domain constraints are in (12-13-14).

\section{Numerical Results}

Both the MILP models (of the G-CVRP-TW and of the E-VRPTW-PR) have been implemented in AMPL and solved by the state of the art solver CPLEX 
12.6 on a PC Intel Core i7, 3.20 GHz, 6GB RAM. Some benchmark instances of [9], with 5,10,15 customers, have been tested. In the G-CVRP-TW, $M$ has been set to the ceiling of 1.2 times the number of vehicles used in the best solution known for the E-VRPTW-PR. In fact, it is worth noting that a low value of $M$ may lead to a harder problem, while a high value, to a poor quality initial solution. In the Phase 3 , the parameters have been tuned in the following way: $r h s_{\min }^{L}=r h s_{\min }^{S}=1, r h s_{\max }^{L}=10, r h s_{\max }^{S}=20$, $k$ step $^{L}=k$ step $^{S}=1, T L^{L}=60$ seconds, $T L^{S}=300$ seconds.

The table shows the comparisons among the MILP model of the E-VRPTWPR, VNSB and TPM. Since they find the same value of $\mu$, we report only one column for it. The columns $T T_{M I L P}, T T_{V N S B}$ and $T T_{T P M}$ display their total TT, respectively; $C P U_{M I L P}, C P U_{V N S B}$ and $C P U_{T P M}$ show the CPU time, in seconds, when each method finds its best solution. The stopping criterion of both, the VNSB and the TPM, given by the CPU time limit, is set to 7,200 seconds as for Cplex. In particular, the cases in which it has been reached by CPLEX obtaining or not a feasible solution are marked by " $\dagger$ " and "-", respectively. The relative percentage gap $(\Delta T T \%)$ between $T T_{V N S B}$ and $T T_{T P M}$ is computed as $\frac{T T_{T P M}-T T_{V N S B}}{T T_{V N S B}} \cdot 100$. The best results are in boldface.

Both TPM and VNSB give the same TT on instances with 5 customers, corresponding to the optimum found by MILP. On instances with 10 customers, in 3 cases, TPM outperforms VNSB with even a case with percentage improvement of $1.66 \%$. While, in one case VNSB outperforms TPM with a percentage improvement of $0.56 \%$, with an average of $0.10 \%$. TPM obtains always solutions better or equal than the MILP and in 8 cases strictly better. On instances with 15 customers, TPM outperforms VNSB in 5 cases vs 4 with a higher average percentage improvement (25.44\%). On 6 over 12 instances, MILP is not able to find even a feasible solution within the CPU time limit, remarking the challenging nature of our problem. Overall, both TPM and VNSB give results by far better than those of the MILP. On average, TPM is more time consuming than VNSB, but it is rewarded by a better solution quality. However, they are by far faster than the MILP.

\section{Conclusions and Future Works}

The efficient routing of a fleet of Electric Vehicles (EVs) was addressed as timeeffective Electric Vehicle Routing Problem with Time Windows and Partial Recharges with the aim of firstly minimizing the total number of EVs used and then, the total Time spent by EVs outside the depot. Such a problem was solved by a Three-Phases Matheuristic (TPM). 


\begin{tabular}{|c|c|c|c|c|c|c|c|c|}
\hline Instance & $\mu$ & $T T_{M I L P}$ & $T T_{V N S B}$ & $T T_{T P M}$ & $\Delta T T \%$ & $C P U_{M I L P}$ & $C P U_{V N S B}$ & $C P U_{T P M}$ \\
\hline C101-5 & 2 & $1,262.84$ & $1,262.84$ & $1,262.84$ & 0 & 3.06 & 0.70 & 0.81 \\
\hline C103-5 & 1 & 987.87 & 987.87 & 987.87 & 0 & 0.16 & 0.29 & 0.28 \\
\hline C206-5 & 1 & $1,296.82$ & $1,296.82$ & $1,296.82$ & 0 & 94.94 & 14.16 & 0.56 \\
\hline C208-5 & 1 & 984.80 & 984.80 & 984.80 & 0 & 4.15 & 1.31 & 1.42 \\
\hline R104-5 & 2 & 196.17 & 196.17 & 196.17 & 0 & 20.68 & 2.43 & 0.07 \\
\hline R105-5 & 2 & 231.59 & 231.59 & 231.59 & 0 & 2.09 & 0.33 & 0.09 \\
\hline R202-5 & 1 & 234.16 & 234.16 & 234.16 & 0 & 19.30 & 1.62 & 0.34 \\
\hline R203-5 & 1 & 287.09 & 287.09 & 287.09 & 0 & 736.64 & 1.81 & 0.31 \\
\hline RC105-5 & 2 & 314.31 & 314.31 & 314.31 & 0 & 58.31 & 94.01 & 215.55 \\
\hline RC108-5 & 2 & 342.32 & 342.32 & 342.32 & 0 & 156.92 & 1.75 & 0.39 \\
\hline RC204-5 & 1 & 264.86 & 264.86 & 264.86 & 0 & $\dagger$ & 49.16 & 0.44 \\
\hline RC208-5 & 1 & 253.17 & 253.17 & 253.17 & 0 & 146.10 & 8.00 & 0.42 \\
\hline Average & & 554.67 & 554.67 & 554.67 & 0 & 703.53 & 14.63 & 18.39 \\
\hline C101-10 & 3 & $2,411.08$ & $2,335.20$ & $2,335.20$ & 0 & $\dagger$ & 183.43 & 545.76 \\
\hline C104-10 & 2 & $1,805.67$ & $1,611.72$ & $1,584.90$ & -1.66 & $\dagger$ & 73.43 & 252.95 \\
\hline C202-10 & 1 & $2,949.77$ & $2,949.77$ & $2,949.77$ & 0 & $\dagger$ & 5.62 & 3.83 \\
\hline C205-10 & 2 & $2,525.77$ & $2,525.77$ & $2,539.88$ & 0.56 & 619.28 & 0.79 & 1.45 \\
\hline R102-10 & 3 & 443.62 & 443.62 & 443.62 & 0 & $6,563.57$ & 33.96 & 0.35 \\
\hline R103-10 & 2 & 351.90 & 347.70 & 347.70 & 0 & $\dagger$ & 93.49 & 12.19 \\
\hline R201-10 & 1 & 536.29 & 536.38 & 536.05 & -0.06 & $\dagger$ & 21.70 & 76.03 \\
\hline R203-10 & 1 & 540.29 & 527.68 & 527.68 & 0 & $\dagger$ & 4.62 & 9.10 \\
\hline RC102-10 & 4 & 571.26 & 571.26 & 571.26 & 0 & $\dagger$ & 59.01 & 24.76 \\
\hline RC108-10 & 3 & 515.11 & 493.23 & 493.23 & 0 & $\dagger$ & 143.71 & 12.73 \\
\hline RC201-10 & 1 & 793.52 & 793.52 & 793.33 & -0.02 & $3,535.97$ & 40.46 & 9.25 \\
\hline RC205-10 & 2 & 618.44 & 611.66 & 611.66 & 0 & $\dagger$ & 37.51 & 4.70 \\
\hline Average & & $1,171.89$ & $1,145.62$ & $1,144.52$ & -0.10 & $6,293.24$ & 58.14 & 79.42 \\
\hline C103-15 & 3 & - & $5,254.93$ & $2,446.99$ & -53.43 & $\dagger$ & 240.46 & 178.86 \\
\hline C106-15 & 3 & $2,356.91$ & $2,173.09$ & $2,173.09$ & 0 & $\dagger$ & 106.30 & 213.12 \\
\hline C202-15 & 2 & $3,936.32$ & $3,664.12$ & $3,599.80$ & -1.76 & $\dagger$ & 139.83 & 61.15 \\
\hline C208-15 & 2 & $3,305.25$ & $2,819.47$ & $2,819.47$ & 0 & $\dagger$ & 591.57 & 72.26 \\
\hline R102-15 & 5 & - & $1,241.80$ & 656.37 & -47.14 & $\dagger$ & 559.52 & 113.75 \\
\hline R105-15 & 4 & 697.13 & 567.82 & 567.82 & 0 & $\dagger$ & 487.02 & 2.23 \\
\hline R202-15 & 2 & 999.72 & 839.74 & 840.99 & 0.15 & $\dagger$ & 349.29 & 58.96 \\
\hline R209-15 & 1 & 771.94 & 621.79 & 640.64 & 3.03 & $\dagger$ & 330.38 & $5,718.31$ \\
\hline RC103-15 & 4 & - & $1,074.90$ & 614.13 & -42.87 & $\dagger$ & 251.55 & 31.81 \\
\hline RC108-15 & 3 & - & 974.88 & 596.17 & -38.85 & $\dagger$ & 756.61 & 288.01 \\
\hline RC202-15 & 2 & - & $3,222.49$ & $1,611.29$ & 50 & $\dagger$ & 444.54 & 71.87 \\
\hline RC204-15 & 1 & - & 685.61 & 685.86 & 0.04 & $\dagger$ & 617.22 & $4,119.53$ \\
\hline Average & & $2,011.21$ & $2,099.79$ & $1,437.72$ & -25.44 & $7,200.00$ & 406.19 & 910.82 \\
\hline
\end{tabular}

Table 1

Comparisons among MILP, VNSB and TPM 
Numerical results, on some benchmark instances, showed that on average TPM outperforms a Variable Neighborhood Search Branching (VNSB), previously proposed. Although on average the computational times are higher, they are justified by a higher solution quality, especially on the bigger size instances. Indeed on the instances with 15 customers TPM outperforms VNSB in 5 cases vs 4 with an average percentage improvement of $25.44 \%$.

Future work may concern the extension of E-VRPTW to include the "regenerative braking", allowing the recuperation of a percentage of the battery consumption during the braking (e.g., in the descents).

\section{References}

[1] Bruglieri, M. , Pezzella, F., Pisacane, O., Suraci, S. (2015). A Variable Neighborhood Search Branching for the Electric Vehicle Routing Problem with Time Windows. Electronic Notes in Discrete Mathematics, 47, 221-228.

[2] Bruglieri, M., Pezzella, F., Pisacane, O., Suraci, S. (2015). A Matheuristic for the Electric Vehicle Routing Problem with Time Windows. eprint arXiv:1506.00211, 2015arXiv150600211B.

[3] Erdogan, S., Hooks, E. M. (2012). A green vehicle routing problem. Transportation Research Part E: Logistics \& Transportation Review, 48, 100114.

[4] Felipe, A., Ortuño, M. T., Righini, G., Tirado, G. (2014). A heuristic approach for the green vehicle routing problem with multiple technologies and partial recharges. Transportation Research Part E: Logistics \& Transportation Review, $71,111-128$.

[5] Fischetti, M., Lodi, A. (2003). Local branching. Mathematical Programming Series B, 98,23-47.

[6] Hansen, P., Mladenovic, N., Urosevic, D. (2006). Variable neighborhood search and local branching. Computers $\& 3$ Operations Research, 33, 3034-3045.

[7] Mladenovic, N., Hansen P. (1997). Variable Neighborhood Search. Computers E) Operations Research, 24 (11), 1097-1100.

[8] Savelsbergh, M. W. P. (1992). The vehicle routing problem with time windows: minimizing route duration. ORSA Journal on Computing, 4(2): 146-154.

[9] Schneider, M., Stenger, A., Goeke, D. (2014). The Electric Vehicle Routing Problem with Time Windows and Recharging Stations. Transportation Science,48 (4), 500-520. 